\title{
Median Filter for Noise Removal using Particle Swarm Optimization
}

\author{
Rajesh Mehra \\ Associate Professor \\ NITTR, Sec-26 \\ Chandigarh, India
}

\author{
Ruby Verma \\ M.E. Student \\ NITTR, Sec-26 \\ Chandigarh, India
}

\begin{abstract}
Adaptive median filter has been an efficient algorithm for salt and pepper noise removal. But, if the noise percentage are very high, adaptive median filter may still remain noise regions in result image. So a Particle swarm optimization based novel and modified adaptive median filter (PSOMF) is proposed. The Proposed filter works in two stages: Noise detection stage and noise filtering stage. Particle swarm optimization is a simple algorithm that seems to be effective for optimizing a wide range of functions. Noise Detection stage works on it. First, a test decides whether or not a given pixel is contaminated by impulse noise. If contaminated, a median fitter is applied. Simulation results show that our method is significantly better than a number of existing techniques in term of image restoration and noise detection, even for noise levels as high as $90 \%$.
\end{abstract}

\section{General Terms}

Image Restoration, Genetic Algorithms, Adaptive Median filter.

\section{Keywords}

Particle Swam Optimization, Impulse Noise, PSNR, IQI, SSIM.

\section{INTRODUCTION}

Noise gets introduced in image via any electrical system used for storage, transmission or processing. Random impulse noise, also known as spike noise or Salt and Pepper, occurs when a pixel takes on either the maximum or minimum value possible generating black or white pixels. It has either 0 or 255 value.

Non-linear median filter (MED) filter is often used to remove noise [1]. Median filter cannot distinguish fine detail from noise. An adaptive median filter (ASMF) preserves fine details as compare to MED filter. For low and medium noise density, the ASMF had a good performance [2]. To improve the performance for the case of high noise density, a modified ASMF filter was introduced. A universal noise removal algorithm works both on Gaussian as well as impulse noise. It gives better results than SD-ROM filter [3]. A rank-order thresholding-based and a soft-switching impulse detector were shown work well under high noise corruption but at the cost of significantly increasing computational complexity [4]. Predictive-based adaptive switching median filter (PASMF) is two stage filter consist of neural network based noise detector [5]. It takes three inputs median filter, rank-ordered logarithmic difference (ROLD), central pixel and median value generates the noise map. PASMF handles noise upto 70 percent. Its performance is degrades with highly corrupted images. In this paper, a new noise removal filter based on genetic algorithm is proposed, which exhibits better results than many other more complicated detectors. The experimental results show that the proposed filter gives significantly better results in terms of Peak-Signal-to-Noise Ratio (PSNR), Image Quality Index (IQI) [6], Structured Similarity Index (SSIM) [7].

Proposed filter works efficiently on highly corrupted images. Experimental results show that this method produces good output, even when tested with the high level of noise.

\section{NOISE MODELS}

To demonstrate the superior performance of the proposed method, experiments have been conducted on two different noise models with different noise densities [8].

\subsection{Noise Model 1}

Noise is modelled as salt-and-pepper impulse noise, where pixels are randomly corrupted by two fixed values, 0 and 255 (for gray-level image), generated with the same probability. That is, for each image pixel at location $(i, j)$ with intensity value $\mathrm{Si}, \mathrm{j}$ the corresponding pixel of the noisy image will be $\mathrm{Xi}, \mathrm{j}$ in which the probability density function of $\mathrm{Xi}, \mathrm{j}$ is,

$$
P(x)= \begin{cases}P / 2 & \text { for } x=0 \\ P / 2 & \text { for } x=225 \\ 1-P & \text { for } x=S_{i, j}\end{cases}
$$

\subsection{Noise Model 2}

Instead of two fixed values, impulse noise could be modeled by two fixed ranges that appear at both ends with a length of $\mathrm{m}$ each, respectively. That is, noise will equal likely be any value in the range of either $[0, \mathrm{~m}]$ or $[255-\mathrm{m}, 255]$. The probability density function of $\mathrm{Xi}, \mathrm{j}$ will be,

$$
P(x)= \begin{cases}P / 2 m & \text { for } 0 \leq x<m \\ P / 2 m & \text { for } x=(225-m)<x \leq 255 \\ 1-P & \text { for } x=S_{i, j}\end{cases}
$$

\section{PROPOSED FILTER}

A novel and modified adaptive median filter, called Particle swarm optimization based median (PSOMF) filter, is proposed and discussed in this section $[9,10]$. A Proposed MF filter is works in two steps. First, a test decides whether or not a given pixel is contaminated by impulse noise. If contaminated, a median fitter is applied. 


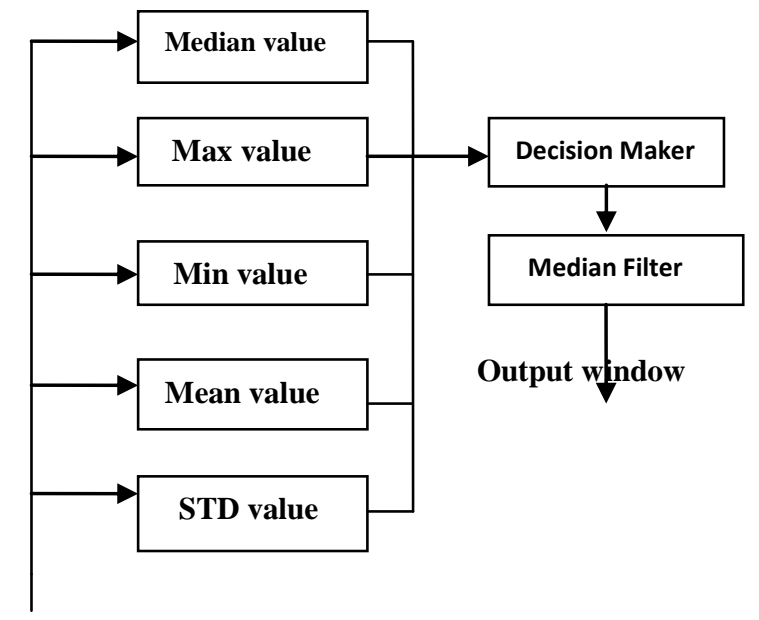

Input window

Fig1. Block diagram of Proposed Filter

\subsection{Decision Maker}

In Proposed filter first step is to train the filter for generating noise map. We initialized 1000 Pixels. In which 500 Pixels are uncorrupted and 500 Pixels are corrupted. Now calculating the value of median value, maximum value, minimum value, mean value and standard deviation of $3 \times 3$ sliding window. These values are become pbest values of features. After 1000 Iterations pbest features taking value of gbest. For finding the corrupted Pixels, comparing the outcomes of FVT (Feature vector Table) with threshold value. If value is above the threshold means corrupted Pixel, if below then uncorrupted Pixel.

$$
\text { NoiseMap }(i, j)= \begin{cases}0 & \text { if output of FVT }<1 \\ 1 & \text { if output of FVT } \geq 1\end{cases}
$$

\subsection{Filtering Stage}

Filter uses a $3 \times 3$ sliding window $\mathrm{W}$, which is located on the corrupted pixel as its centre $\left(\mathbf{X}_{\mathbf{i}, \mathbf{j}}\right)$. Filter simply calculates the median of uncorrupted neighboring pixels and replaces the value of central pixel by the computed median value [11].

\section{EXPERIMENTAL OUTCOMES}

The performance of the filtering process is measured using Four different noise models. The PSNR, IQI (Image Quality Index) values are measured with different noise percentage.

$$
\text { PSNR }=10 \log 10\left(255^{2} / \mathrm{MSE}\right)
$$

Where MSE $=\sum m \sum n[O(m, n)-R(m, n)]^{2} /(\mathrm{MN})$

Where $O$ is the original image, $R$ is the restored image, MSE is the mean squared error, $\mathrm{M} \times \mathrm{N}$ is the size of the image. Here Size of gray scale image is $512 \times 512$. This Image quality index models is a combination of three different factors: loss of correlation, luminance distortion, and contrast distortion [6].

$$
\mathrm{IQI} w=\operatorname{Corr}(O w, R w) \times \operatorname{Lum}(O w, R w) \times \operatorname{Cont}(O w, R w)
$$

The local quality index IQI $w$ is computed within a particular sliding window $\mathrm{W} O w$ and $R w$ represent the sliding window of original and restored images, respectively $[8,15]$. The dynamic range of IQI is $[-1,1]$. The best value 1 is achieved if and only if restored image $R$ is equal to the original image $O$. Black dots in Image quality map shows dissimilarity in original and restored image while white dots shows similarity. Light colour map shows excellent result.

The complete definition of similarity measures three components Luminance, contrast and Structure are combined to yield an overall similarity measure. The similarity measure to satisfy the following conditions.

1) Symmetry: $S(x, y)=S(y \cdot x)$

2) Boundedness: $S(x, y) \leq 1$.

3) Unique maximum: $S(x, y)=1$ if and only if $x=y$.

Table 1. PSNR values with Boat Image (Noise Model 1)

\begin{tabular}{|c|c|c|c|c|c|}
\hline $\begin{array}{c}\text { NOISE } \\
\%\end{array}$ & $\begin{array}{c}\text { MED } \\
{[5]}\end{array}$ & $\begin{array}{c}\text { AMED } \\
{[5]}\end{array}$ & $\begin{array}{l}\text { Kaliraj et } \\
\text { al.[5] }\end{array}$ & $\begin{array}{c}\text { PASMF } \\
{[5]}\end{array}$ & $\begin{array}{c}\text { Proposed } \\
\text { MF }\end{array}$ \\
\hline 90 & 8.38 & 10.32 & 22.51 & 22.72 & 33.2169 \\
\hline 80 & 12.71 & 15.46 & 24.59 & 25.01 & 33.8129 \\
\hline 70 & 17.37 & 20.96 & 24.59 & 26.90 & 35.0247 \\
\hline 60 & 20.43 & 25.03 & 27.83 & 28.25 & 36.3066 \\
\hline 50 & 21.72 & 27.13 & 29.62 & 29.55 & 36.4634 \\
\hline 40 & 22.60 & 28.52 & 30.78 & 30.91 & 38.4692 \\
\hline 30 & 23.21 & 30.40 & 32.37 & 32.55 & 39.8055 \\
\hline 20 & 24.06 & 32.21 & 34.53 & 34.91 & 41.5329 \\
\hline 10 & 24.91 & 33.91 & 37.80 & 38.20 & 44.6177 \\
\hline
\end{tabular}

To verify the advantage and effectiveness of our filtering algorithm, a gray-scale Lena, Boat image whose size is $512 \times$ 512 are selected. The Canny operator is used for calculating the edge maps [12]. Edge map of original and corrupted image are shown in every case. To further check the performance of the proposed algorithm, a one-dimensional signal (200 row) of original and corrupted image has been shown in the form of a graph. Figure 2 and 3 shows the image results based on noise model 1 . Figure 3 and 4 shows the image results based on model 2. 
Table 2. SSIM Values Obtained For Boat Image Based On Noise Model 1

\begin{tabular}{|c|c|c|c|c|c|}
\hline NOISE \% & MED[5] & AMED[5] & Kaliraj et al.[5] & PASMF[5] & Proposed MF \\
\hline 90 & 0.167 & 0.316 & 0.909 & 0.913 & 0.9646 \\
\hline 80 & 0.482 & 0.666 & 0.943 & 0.948 & 0.9704 \\
\hline 70 & 0.758 & 0.885 & 0.961 & 0.967 & 0.9772 \\
\hline 60 & 0.868 & 0.953 & 0.973 & 0.976 & 0.9825 \\
\hline 50 & 0.899 & 0.970 & 0.980 & 0.982 & 0.9861 \\
\hline 40 & 0.916 & 0.978 & 0.986 & 0.989 & 0.9920 \\
\hline 30 & 0.927 & 0.986 & 0.990 & 0.991 & 0.9940 \\
\hline 20 & 0.939 & 0.990 & 0.994 & 0.995 & 0.9963 \\
\hline 10 & 0.949 & 0.993 & 0.997 & 0.999 & 0.9983 \\
\hline
\end{tabular}

Table 3. IQI Values Obtained For Boat Image Based On Noise Model 1

\begin{tabular}{|c|c|c|c|c|c|}
\hline NOISE $\%$ & MED[5] & AMED[5] & Kaliraj et al.[5] & PASMF[5] & Proposed MF \\
\hline 90 & 0.023 & 0.064 & 0.553 & 0.559 & 0.9503 \\
\hline 80 & 0.104 & 0.258 & 0.691 & 0.699 & 0.9582 \\
\hline 70 & 0.251 & 0.505 & 0.772 & 0.779 & 0.9658 \\
\hline 60 & 0.347 & 0.659 & 0.834 & 0.839 & 0.9731 \\
\hline 50 & 0.389 & 0.736 & 0.876 & 0.879 & 0.9784 \\
\hline 40 & 0.406 & 0.788 & 0.907 & 0.911 & 0.9860 \\
\hline 30 & 0.416 & 0.835 & 0.939 & 0.941 & 0.9896 \\
\hline 20 & 0.426 & 0.865 & 0.961 & 0.965 & 0.9934 \\
\hline 10 & 0.433 & 0.875 & 0.982 & 0.984 & 0.9968 \\
\hline
\end{tabular}

Table 4. PSNR Values Obtained For Lena Image Based On Noise Model 2

\begin{tabular}{|c|c|c|c|c|}
\hline NOISE \% & MED[5] & AMED[5] & PASMF[5] & Proposed MF \\
\hline 90 & 08.76 & 10.64 & 26.592448 & 33.1224 \\
\hline 80 & 13.33 & 16.05 & 29.097967 & 34.0440 \\
\hline 70 & 18.53 & 21.92 & 31.387784 & 35.3951 \\
\hline 60 & 22.14 & 27.23 & 32.956618 & 36.0677 \\
\hline 50 & 23.65 & 30.09 & 34.476593 & 37.0928 \\
\hline 40 & 24.51 & 31.79 & 36.290139 & 39.3405 \\
\hline 30 & 25.48 & 33.85 & 38.310070 & 39.9616 \\
\hline 20 & 27.00 & 35.97 & 40.202637 & 42.1757 \\
\hline 10 & 28.47 & 38.18 & 44.226346 & 45.0307 \\
\hline
\end{tabular}

In table 1, 2 and 3 Proposed filter was compared with standard median filter (MED), adaptive median filter (AMED), Kaliraj et al.[13] and Predictive-based adaptive switching median filter (PASMF) on PSNR, IQI, SSIM Value. In table 4, 5 and 6 proposed filter was compared with standard median filter (MED), adaptive median filter (AMED) and Predictive-based adaptive switching median filter (PASMF). In the last image of every figure image quality map of restored image is shown [14]. Black dots in Image Quality map shows dissimilarity of original image with restored image. Light Quality map shows resemblance of original and restored image. 
Table 5. IQI Values Obtained For Lena Image Based On Noise Model 2

\begin{tabular}{|c|c|c|c|c|}
\hline NOISE $\%$ & MED [5] & AMED[5] & PASMF[5] & PSOMF \\
\hline 90 & 0.022 & 0.057 & 0.658 & 0.951 \\
\hline 80 & 0.123 & 0.271 & 0.779 & 0.960 \\
\hline 70 & 0.325 & 0.549 & 0.845 & 0.973 \\
\hline 60 & 0.463 & 0.703 & 0.888 & 0.978 \\
\hline 50 & 0.518 & 0.777 & 0.917 & 0.984 \\
\hline 40 & 0.538 & 0.827 & 0.942 & 0.991 \\
\hline 30 & 0.553 & 0.865 & 0.961 & 0.995 \\
\hline 20 & 0.5632 & 0.887 & 0.973 & 0.997 \\
\hline 10 & 0.569 & 0.894 & 0.989 & \\
\hline
\end{tabular}

Table 6. SSIM Values Obtained For Lena Image Based On Noise Model 2

\begin{tabular}{|c|c|c|c|c|}
\hline NOISE \% & MED[5] & AMED[5] & PASMF[5] & PSOMF \\
\hline 90 & 0.2041 & 0.3432 & 0.94638 & 0.9614 \\
\hline 80 & 0.5413 & 0.7137 & 0.96972 & 0.9687 \\
\hline 70 & 0.8201 & 0.9132 & 0.98219 & 0.9803 \\
\hline 60 & 0.9162 & 0.9731 & 0.98759 & 0.9843 \\
\hline 50 & 0.9404 & 0.9863 & 0.99125 & 0.9893 \\
\hline 40 & 0.9506 & 0.9914 & 0.99424 & 0.9938 \\
\hline 30 & 0.9609 & 0.9946 & 0.99638 & 0.9946 \\
\hline 20 & 0.9714 & 0.9963 & 0.99861 & 0.9974 \\
\hline 10 & 0.9791 & 0.9988 & 0.99899 & 0.9985 \\
\hline
\end{tabular}
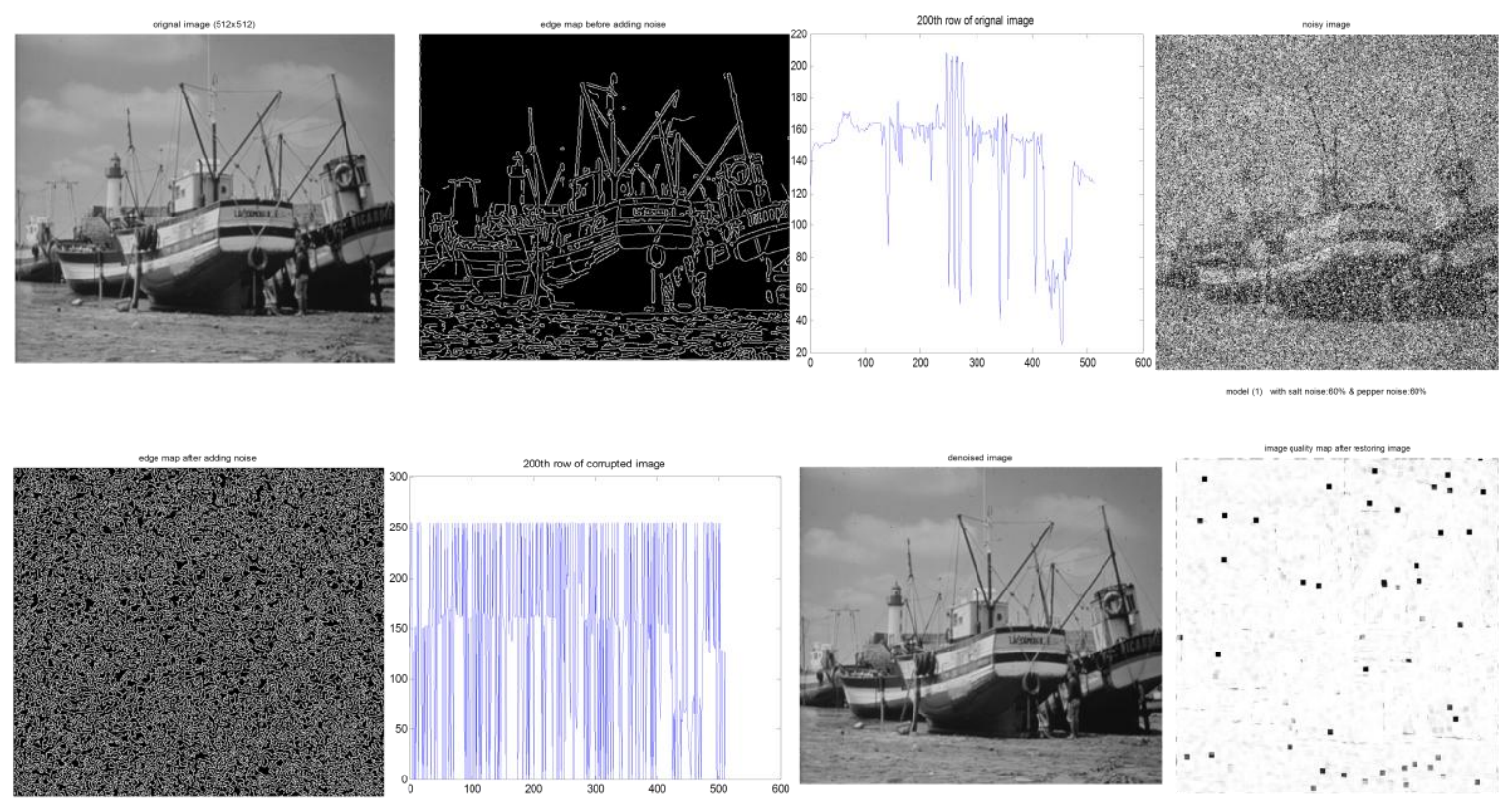

Fig 2. Boat image corrupted by $60 \%$ noise based on noise model 1 

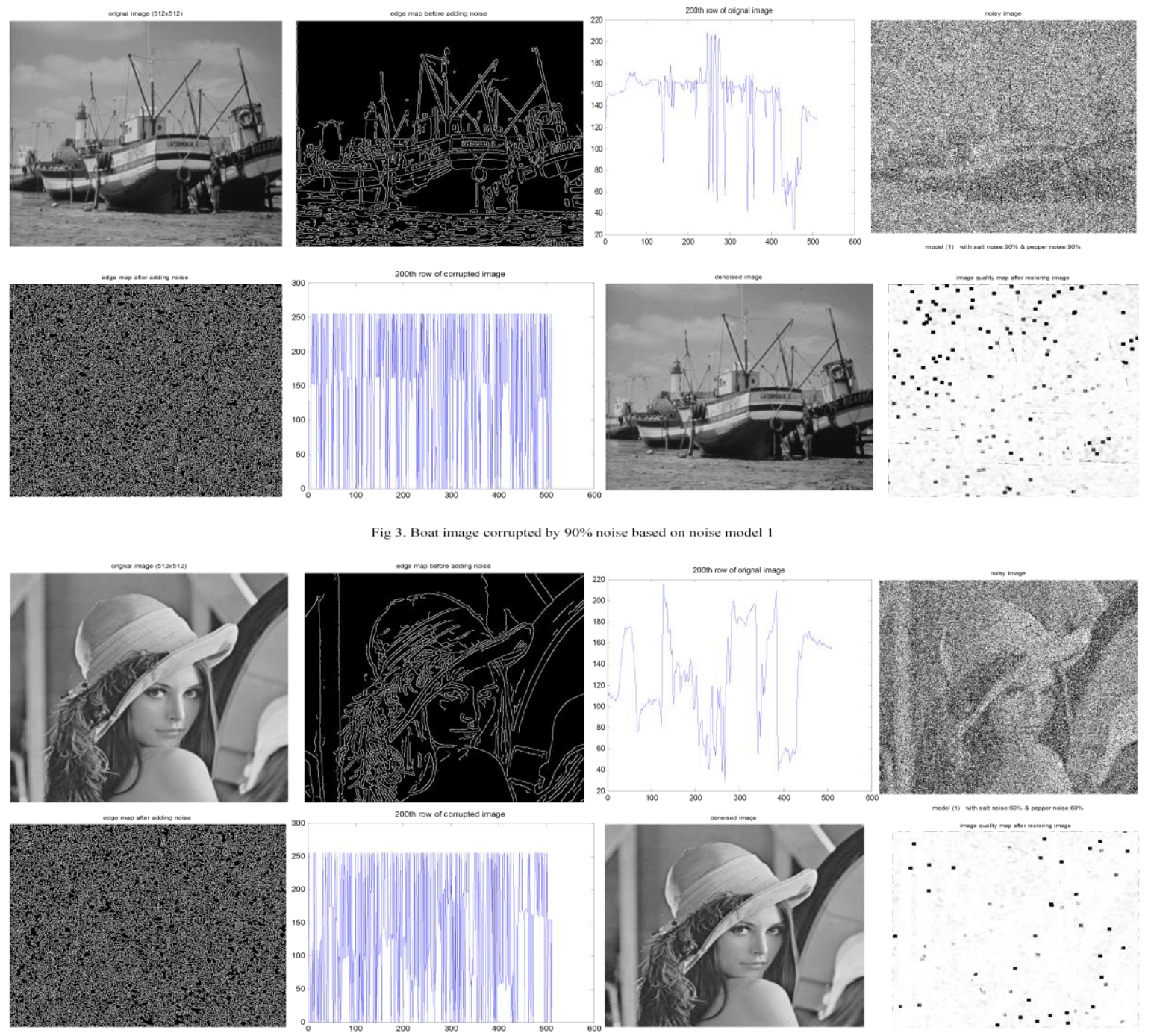

Fig 4. Lena image corrupted by $60 \%$ noise based on noise model 2
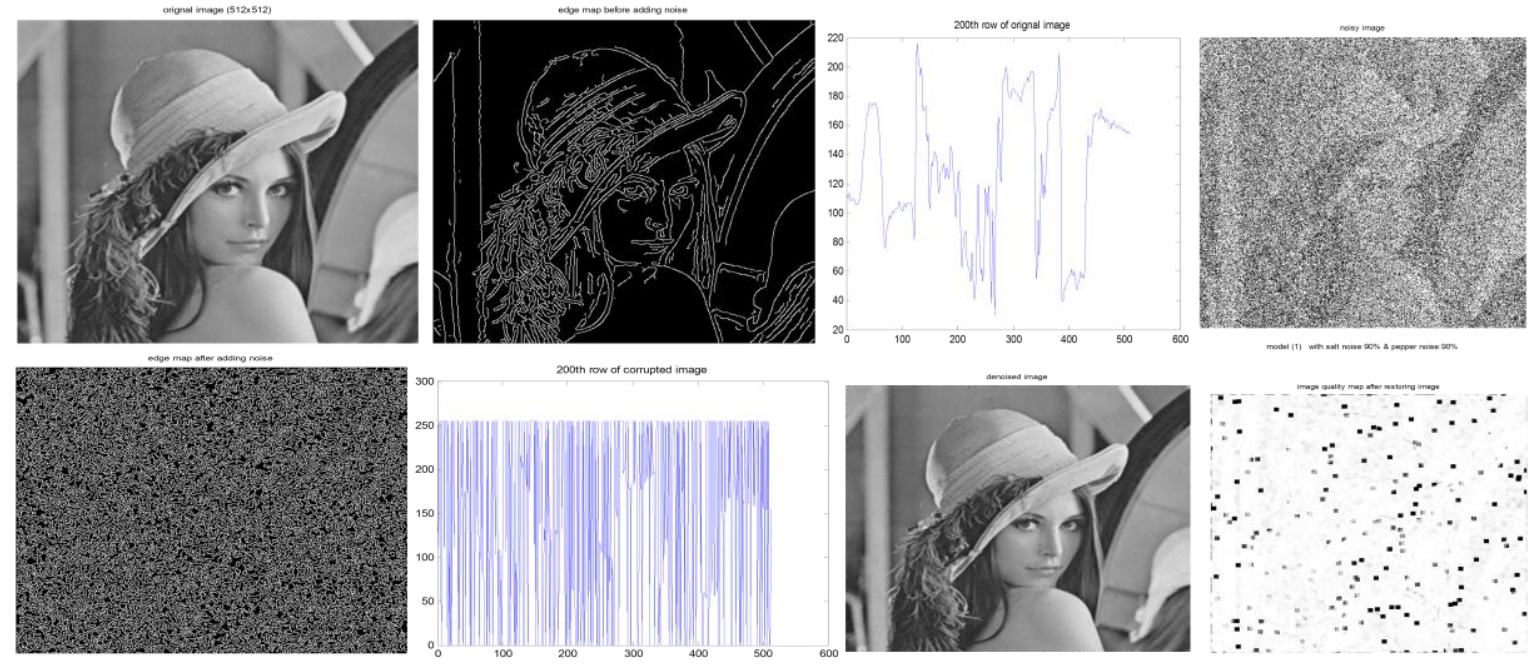

Fig 5. Lena image corrupted by $90 \%$ noise based on noise model 2 


\section{CONCLUSION}

This paper presents a novel technique to remove salt and pepper noise from highly corrupted images. To demonstrate the superior performance of the proposed method, extensive experiments have been conducted on a variety of standard test images to compare with many other well known techniques. Experimental results show that this method always produces good output, even when tested with the high level of noise. Both the simulation and computational complexity analysis show that the proposed method is better than existing standard median filter (MED), adaptive median filter (AMED), Kaliraj et al. filter [15] and Predictive-based adaptive switching median filter (PASMF). Some points can be discussed for further research.

1. Image Enhancement Factor (IEF) is not improved in noise model 2 in comparison to PASMF filter.

2. Proposed filter Tested on gray scale images only.

\section{REFERENCES}

[1] R. C.Gonzalez, R. E. Woods “ Digital Image Processing. $3^{\text {rd }} \quad$ edn. Prentice-Hall, ” Englewood Cliffs, 2009.

[2] S. Akkoul, R. Ledee, R. Leconge, R. Harba, "A new adaptive switching median filter," IEEE Signal Processing Letters, vol.17, pp.587-590, 2010.

[3] B. Deka and D. Baishnab, "A Linear Prediction Based Switching Median Filter for the Removal of Salt and Pepper Noise from Highly Corrupted Image," IEEE conference on Computational Intelligence and signal processing, pp. 99-102, 2012.

[4] K-K.Ma, H.L. Eng: "Noise Adaptive Soft-Switching Median Filter," IEEE Transaction on image processing, vol.10, pp.242-251, February 2001.

[5] M.S. Nair, V. Shankar: "Predictive-based adaptive switching median filter for impulse noise removal using neural network-based noise detector", Springer-london Signal, Image and Video processing, vol. 7, pp.10411070, April 2012.

[6] Z. Wang, A.C. Bovik,: "A universal image quality index". IEEE Signal Processing. Letters, vol. 9, pp.8184 , March 2002.

[7] Z. Wang, A.C. Bovik, H. R.Sheikh,: "Image quality assessment: from error visibility to structural similarity". IEEE Transaction on Image Processing, vol. 13, pp. 600-612, 2004.

[8] P. E. Ng, K.-K Ma,: "A switching median filter with boundary discriminative noise detection for extremely corrupted images". IEEE Transaction on Image Processing, vol. 15, pp.1506-1516, June 2006.

[9] J. Kennedy , R. Eberhart ,: "Particle Swarm Optimization," Proceedings of IEEE Conference on Neural Networks, pp. 1942-1948,1995.

[10] R. Eberhat and Y. Shi, "Particle Swarm Optimization : Development, Applications and Resources," IEEE conference on Evolutionary computation, vol. 1, pp.8186, 2001.

[11] S.J. Ko, Y.H. Lee, "Center weighted median filters and their application to image enhancement," IEEE Transaction on Circuits Systems, Vol. 38, pp.984993,1991.

[12] Rupinder Verma and Rajesh Mehra, "Area Efficient FPGA Implementation of Sobel Edge Detector for Image Processing Applications," International Journal of Computer Applications, Vol. 56, No. 16, pp. 7-11, October 2012.

[13] G. Kaliraj, S. Baskar,: "An Efficient approach for the removal of impulse noise from the corrupted image using neural network based impulse detector," Image and Vision Computing, Vol. 28, pp.458-466, July 2009

[14] Payal Agarwal and Rajesh Mehra, "High Speed CT Image Reconstruction using FPGA," International Journal of Computer Applications, Vol. 22, No. 4, pp. 7 10, May 2011.

[15] Sugreev Kaur and Rajesh Mehra, "High Speed and Area Efficient 2D DWT Processor Based Image Compression,": An International Journal on Signal And Image Processing, Vol.1, No. 2, pp. 22- 31, December 2010 .

\section{AUTHOR'S PROFILE}

Dr. Rajesh Mehra: Dr. Mehra is currently associated with Electronics and Communication Engineering Department of National Institute of Technical Teachers' Training \& Research, Chandigarh, India since 1996. He has received his Doctor of Philosophy in Engineering and Technology from Panjab University, Chandigarh, India in 2015. Dr. Mehra received his Master of Engineering from Panjab Univeristy, Chandigarh, India in 2008 and Bachelor of Technology from NIT, Jalandhar, India in 1994. Dr. Mehra has 20 years of academic and industry experience. He has more than 320 papers to his credit which are published in refereed International Journals and Conferences. Dr. Mehra has guided $70 \mathrm{ME}$ thesis and he is also guiding 02 independent $\mathrm{PhD}$ scholars in his research areas. He has also authored one book on PLC \& SCADA. He has developed 06 video films in VLSI area. His research areas are Advanced Digital Signal Processing, VLSI Design, FPGA System Design, Embedded System Design, and Wireless \& Mobile Communication. Dr. Mehra is member of IEEE and ISTE.

Ms. Ruby Verma: Ms. Ruby is currently pursuing M.E from National Institute of Technical Teachers Training \& Research Chandigarh, India. She has completed B.Tech from R.B.S. college Uttar Paradesh Technical university, Agra (U.P.). She is having six years of teaching experience in Doon Institute of Engineering \& Technology DIET, Dehradun (U.K.). Ms. Ruby's interest areas are Image processing, VLSI, Wireless Communication and Digital Electronics 